\title{
Modeling and Analysis of LPI Radar Signal
}

\author{
Ramya Vellanki ${ }^{1}$, K.Satish Babu ${ }^{2}$ \\ ${ }^{I}$ (Embedded Systems, ATRI, India) \\ ${ }^{2}$ (ECE, ATRI, India)
}

\begin{abstract}
Radar Emitter Signal recognition is one of the key procedures of signal processing in Electronic Warfare (EW) Receiver. As there is an intense growth in the activities of modern Electronic Warfare, advanced radars are increasing and becoming main component of radars gradually. These modern radars called Low Probability of Intercept (LPI), use different complex waveforms to mask their presence from the enemy while accomplishing their mission. These waveforms cannot be detected by the use of traditional recognition algorithms and methods thus posing a major challenge to the Electronic Warfare designer. This paper presents simulation of different types of LPI signals, generation of those signals and development of Time Frequency Algorithm to analyze those signals.
\end{abstract}

Keywords: Cross terms, ELINT (Electronic Intelligence), EW (Electronic Warfare), Intercept Receiver, LPI (Low Probability of Intercept).

\section{Introduction}

On the modern battlefield, radar system face serious threats from passive intercept receivers such as Electronic Attack (EA) and Anti Radiation Missiles (ARMs). To perform necessary target detection and tracking and simultaneously hide themselves from enemy attack, radar systems should be Low Probability of Intercept (LPI). Low Probability of Intercept (LPI) radars have the property of low power, wide bandwidth, frequency variability, or other design attributes to make them difficult to be detected or identified by passive intercept receiver devices such as Electronic Support (ES) or Electronic Intelligence (ELINT) receivers ${ }^{[1]}$. LPI radars attempt to provide the detection of targets at longer ranges than intercept receivers can accomplish detection of the radar. The success of LPI radar is measured by how hard it is for them to be detected.

The Electronics Warfare (EW) scenario has become so complex today and many users of Radar today are specifying a Low Probability of Intercept (LPI) as an important tactical requirement. The LPI radar transmitter makes use of sophisticated frequency and phase modulation to spread the signal bandwidth making the signal hard to intercept ${ }^{[2]}$. The receiver makes use of the appropriate matched filter so that the radar performance is similar to that of traditional pulsed radar radiating the same amount of average power.

\section{LPI Radar Signal Generation}

There are several LPI radar techniques available to the modern radar designer in various combinations, depending on the application. Reducing the radar's peak effective radiated power (ERP) by using some form of pulse compression technique is the most common LPI radar technique ${ }^{[1]}$. The objective is to spread the radar's signal over a wide bandwidth and a period of time.

The most common modulation techniques available to provide LPI features are Barker codes and Polyphase Codes (Frank, P1-P4).

\subsection{Barker Codes}

The binary Barker sequences are finite length, discrete time sequences with constant magnitude and a phase of either $\varphi_{k}=0$ or $\varphi_{k}=\pi$. It is a finite length sequence $A=\left[a_{0}, a_{1} \ldots a_{n}\right]$ of +1 's and -1 's of length $n \geq 2$ such that the aperiodic autocorrelation coefficients (or side lobes) ${ }^{[1]}$

$$
r k=\sum_{j=1}^{n-k} a_{j} a_{j+k}
$$

satisfies $\left|\mathrm{r}_{\mathrm{k}}\right| \leq 1 \quad$ for $\mathrm{k} \neq 0$ and similarly $\mathrm{r}_{\mathrm{k}}=\mathrm{r}_{-\mathrm{k}}$;

A binary Barker sequence has elements $a_{i} \in\{-1,+1\}$, which are only known for lengths $\mathrm{N}_{c}=2,3,4,5$, 7,11 , and 13 . The Barker codes are the most frequently used binary code since they result in an ambiguity function with side lobe levels at zero Doppler, not higher than $1 / \mathrm{N}_{\mathrm{c}}$ relative to a main lobe of level 1 . In fact, due to this property, Barker codes are often called perfect codes.

The sequences where a +1 i.e. a $0^{0}$ phase is represented by a + and a -1 i.e. $180^{\circ}$ phase is represented by $\mathrm{a}-$. Binary Barker sequences with lengths greater than 13 , with $\mathrm{N}_{\mathrm{c}}$ odd, do not exist.

This radar has unique collection of properties such as good auto correlation properties, low side lobes, offer covertness to the radar signal making its detection difficult with conventional intercept receivers. 
An LPI Radar signal modulated using seven bit Barker sequence is shown in Fig 1. From the figure we can observe there are three phase changes one after three cycles, the second one after five cycles and third one after six cycles, since the seven bit barker sequence i.e. +++--+- has three phase changes at those locations.

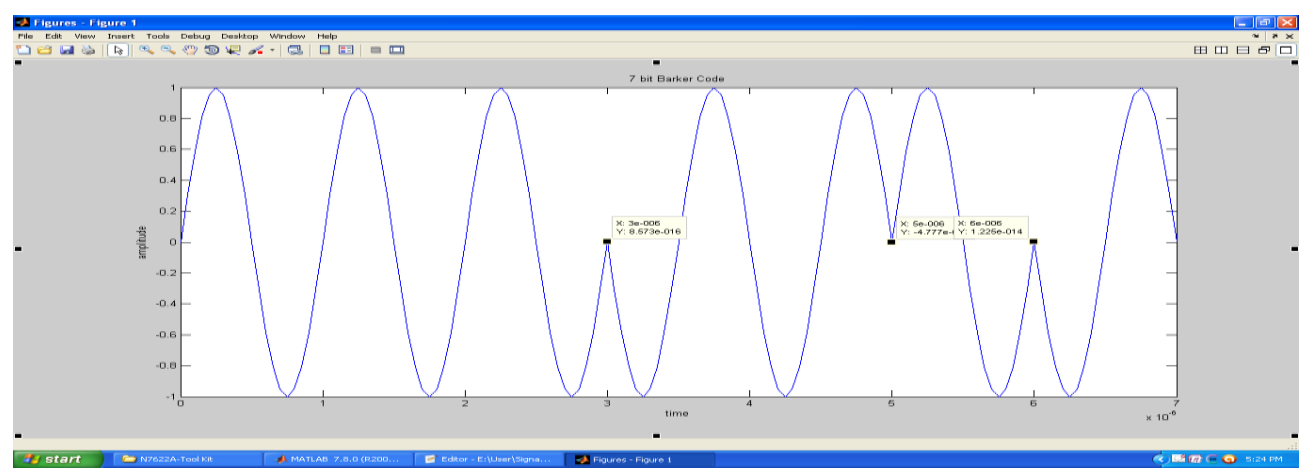

Figure 1: Seven bit Barker Code

The phase versus time graph for seven bit barker code is shown in Fig 2 below:

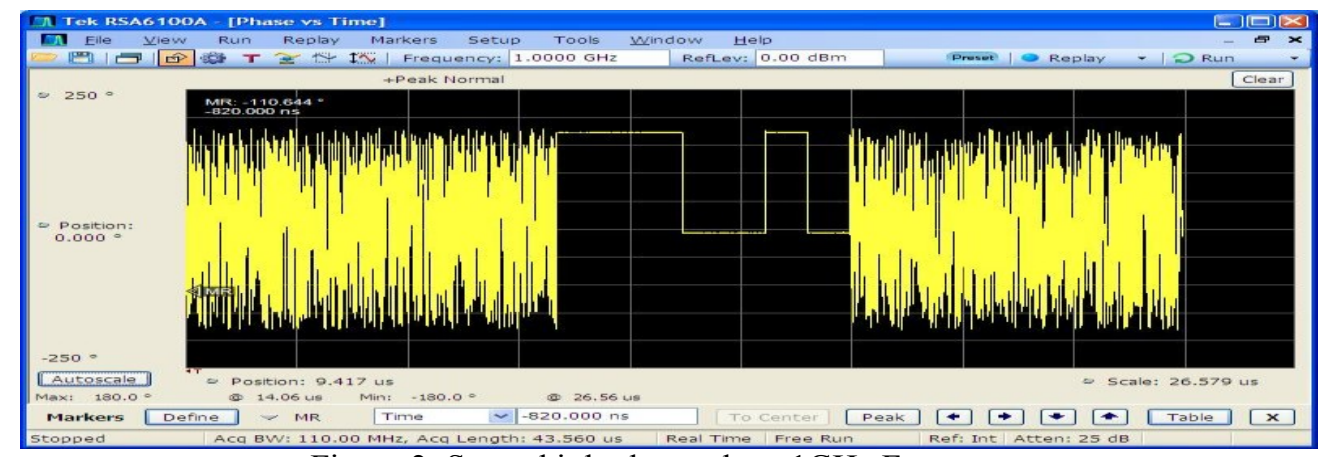

Figure 2: Seven bit barker code at $1 \mathrm{GHz}$ Frequency

\subsection{Poly Phase Codes}

Poly phase sequences are finite length, discrete time complex sequences with constant magnitude but with a variable phase $\phi_{k}{ }^{[1]}$. Polyphase coding refers to phase modulation of the CW carrier, with a poly phase sequence consisting of number of distinct phases. Frank, P1, P2, P3, P4 Codes comes under Polyphase codes.

\subsubsection{Frank Code}

The Frank Code is derived from a step approximation to a linear frequency modulation waveform using $\mathrm{M}$ frequency steps and $\mathrm{M}$ samples per frequency. The frank code has a length or processing gain of $\mathrm{Nc}=\mathrm{M}^{*} \mathrm{M}$.If $i$ is the number of the sample in a given frequency and $\mathrm{j}$ is the number of the frequency, the phase of the $i^{t h}$ sample of the $j^{\text {th }}$ frequency is:

$$
\phi_{i, j}=2 \pi / M[(i-1)(j-1)]
$$

where $i=1,2,3, \ldots, \mathrm{M}$ and $\mathrm{j}=1,2,3 \ldots, \mathrm{M}$.

The Simulation results for the Frank Code are shown in Fig 3.

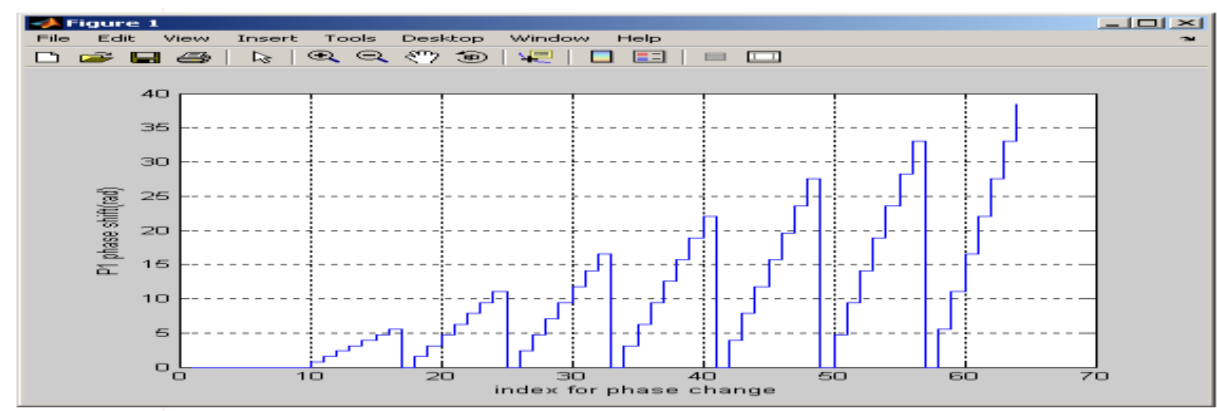

Figure 3: Frank Code phase values for $\mathrm{M}=8$ 


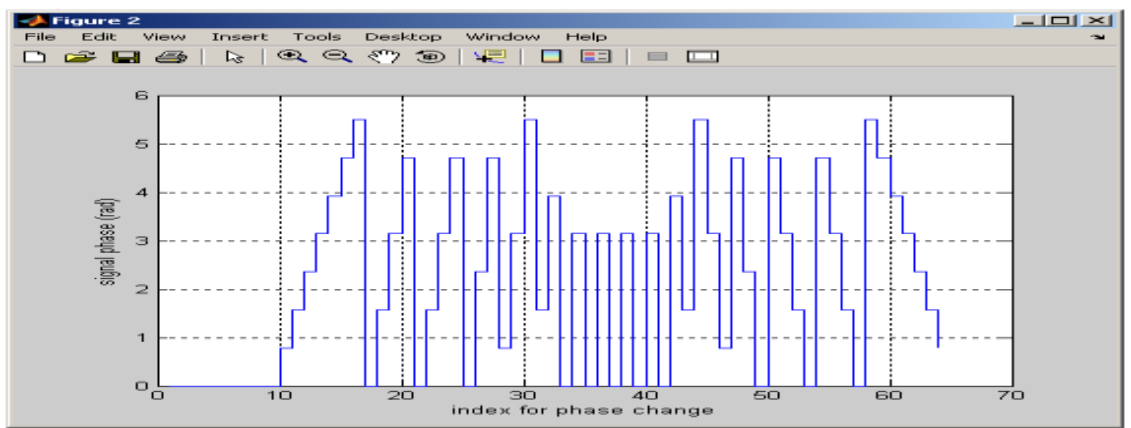

Figure 4: Frank Code values for $\mathrm{M}=8$ showing signal phase modulo $2 \pi$

\subsubsection{P1 Code}

The P1 code is also generated using a step approximation to a linear frequency modulation waveform. In this code, $M$ frequency steps and $\mathrm{M}$ samples per frequency are obtained from the waveform using double sideband detection with the local oscillator at band center. The length of the resulting code or compression ratio is $\mathrm{Nc}=\mathrm{M} \mathrm{XM}$.If $i$ is the number of the sample in a given frequency and $\mathrm{j}$ is the number of the frequency, the phase of the $i^{\text {th }}$ sample of the $j^{\text {th }}$ frequency is:

$\phi_{i, j}=-\pi / M[M-(2 j-1)][(j-1) M+(i-1)]$

where $i=1,2,3 . ., \mathrm{M}, \mathrm{j}=1,2,3 \ldots ., \mathrm{M}$ and $\mathrm{M}=1,2,3 \ldots$

The Simulation results for the Frank Code are shown in Fig 5:
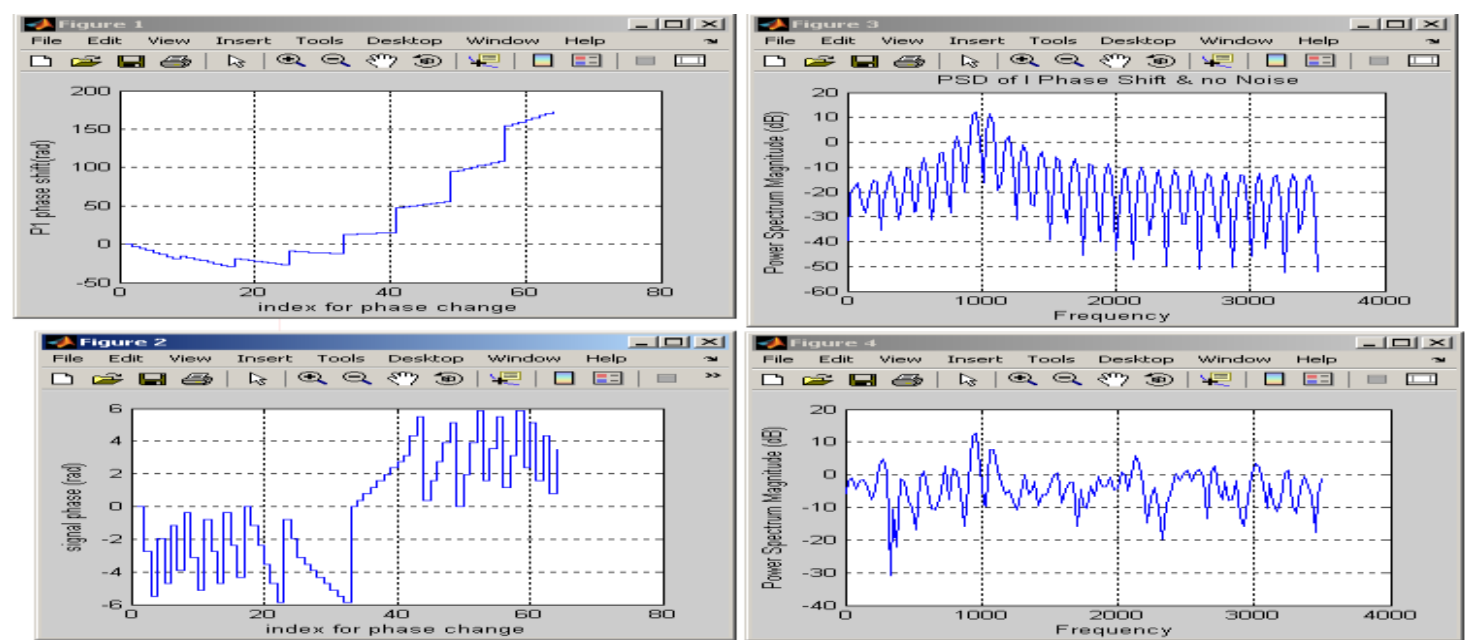

Figure 5: $\mathrm{P} 1$ code phase values for $\mathrm{M}=8$

\subsubsection{P2 Code}

For the P2 code $\mathrm{M}$ even, the phase increments within each phase group is the same as the P1 code, except that the starting phases are different. The $\mathrm{P} 2$ code also has a length or compression ratio of $\mathrm{NC}=\mathrm{M}^{*} \mathrm{M}$ .The $\mathrm{P} 2$ code is given by:

$\phi_{i, j}=-\pi / 2 M[2 i-1-M][2 j-1-M]$

Where $i=1,2,3, \ldots, M$ and $j=1,2,3 \ldots ., M$ and where $M=2,4,6 \ldots$

The Simulation results for the Frank Code are shown in Fig 6: 

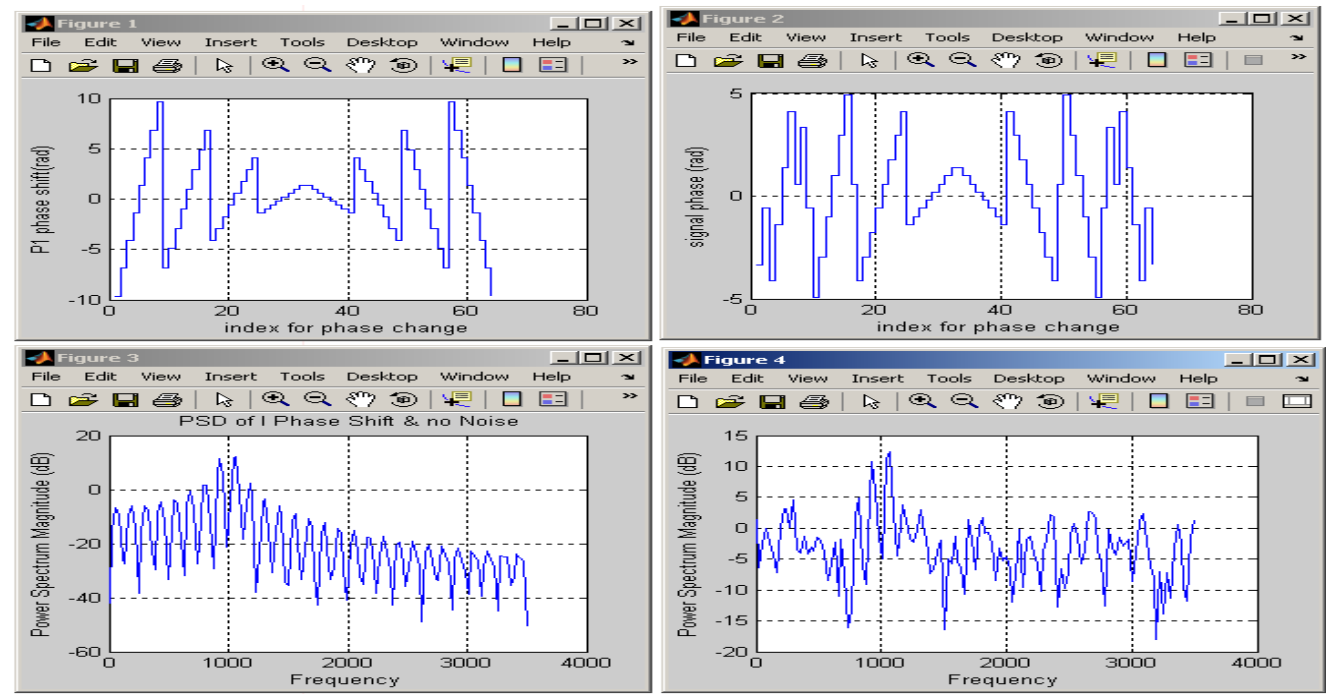

Figure 6: $\mathrm{P} 2$ code phase values for $\mathrm{M}=8$

\subsubsection{P3 Code}

The P3 code is conceptually derived by converting a linear frequency modulation waveform to base band, by synchronous oscillator on one end of the frequency sweep (single side band detection) and sampling the I and Q video at Nyquist rate. The phase of the $i^{\text {th }}$ sample of the P3 code is given by:

$\phi_{i}=-\pi / N_{c}(i-1)^{2}$

where $\mathrm{i}=1,2 \ldots \mathrm{Nc}$ and $\mathrm{Nc}$ is the compression ratio.

The Simulation results for the Frank Code are shown in Fig 7:
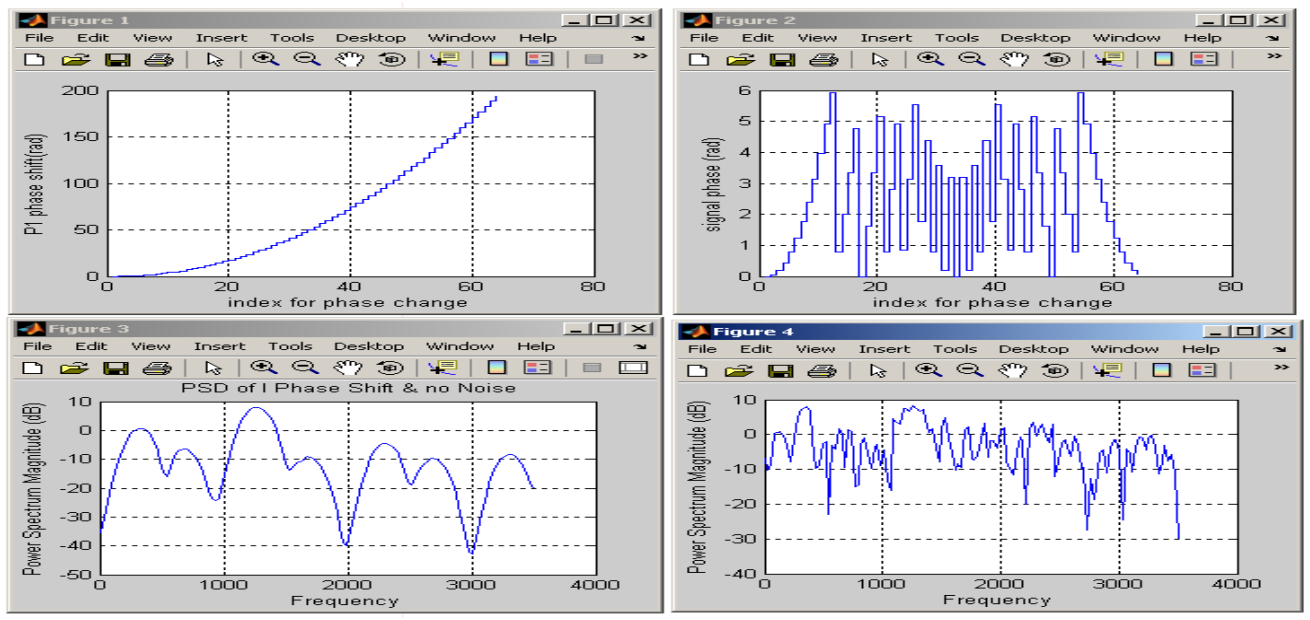

Figure 7: $\mathrm{P} 3$ code phase values for $\mathrm{M}=8$

\subsection{5. $\mathrm{P} 4$ code}

The P4 code is conceptually derived from the same linear frequency modulation waveform as the P3 code with a different phase equation given by,

The phase sequence of a P4 signal is described by:

$$
\phi_{i}=-\pi(i-1)^{2} / N_{c}-\pi(i-1)
$$

For $i=1$ to $\mathrm{Nc}$ where $\mathrm{Nc}$ is the pulse compression ratio.

The Simulation results for the Frank Code are shown in Fig 8: 

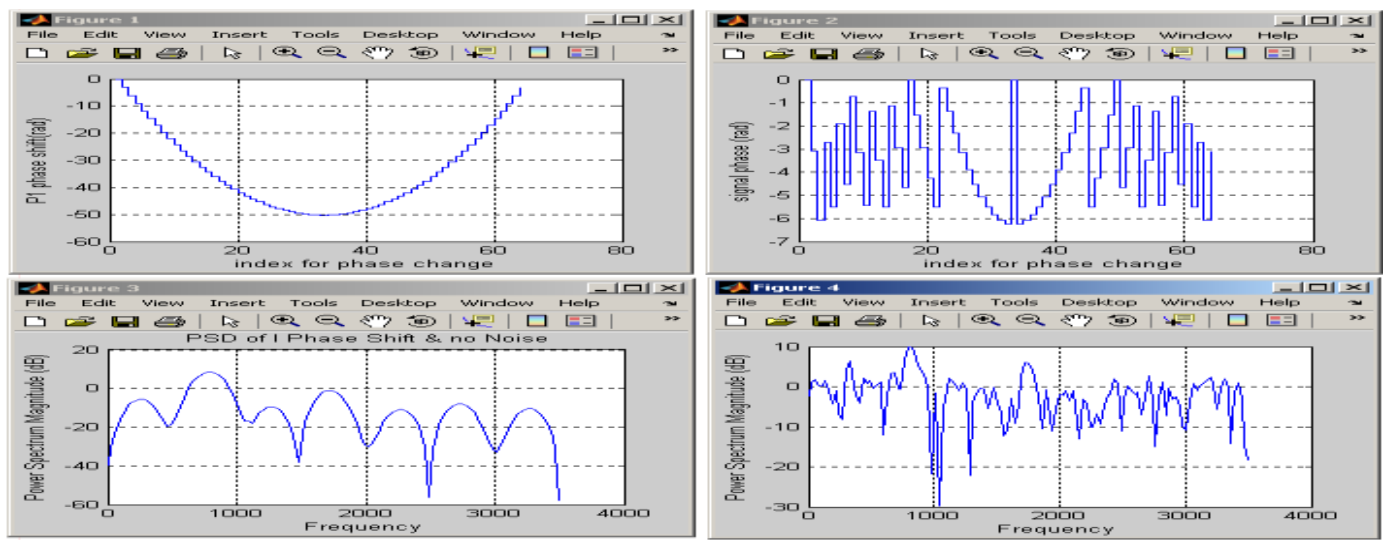

Figure 8: $\mathrm{P} 4$ code phase values for $\mathrm{M}=8$

\section{Design Approach for Analysis of LPI Waveforms}

To analyze the LPI signal Joint Time Frequency analysis (JFTA) technique will be used. One method of visualizing a signal is in the time domain. This representation often plots the signal amplitude as a function of time. Then represent signal in frequency domain based on a variation of the Fourier Transform to get the spectral components. The Fourier Transform is a very useful concept for stationary signals. Many signals encountered in real world situations have frequency content that changes over time. To represent signals of this nature, Joint time frequency transforms were developed which characterized the exact behavior of the time varying frequency content of the signal. Joint Time Frequency Analysis (JFTA) is a very powerful tool for removing noise and interference from a signal. Time Frequency methods are used for determining range of target, which is a function of time, and the target speed, which is the function of frequency. For the above reasons, we can apply a type of Joint Time Frequency Analysis (JFTA).

\subsection{Wigner Ville Distribution (WVD)}

The Wigner Ville Distribution (WVD) is a two-dimension function describing the frequency content of a signal as a function of time. Using the WVD, frequency and time changes in most of the LPI radar signals can be identified. The WVD of discrete input signal $\mathrm{x}(\mathrm{t})$ is defined as:

$$
w(l, w)=2 \sum_{n=-N+1}^{N-1} x(l+n) x^{*}(l-n) w(n) w(-n) e^{-j 2 w n}
$$

where $\mathrm{x}(\mathrm{n})$ is an input signal

$l$ is the time variable,

$\omega$ is the angular frequency variable,

* indicates the complex conjugate

$\mathrm{w}(\mathrm{n})$ is a length of $2 \mathrm{~N}-1$ real window function with $\mathrm{w}(0)=1$.

\subsection{Choi-Williams Distribution (CWD)}

The Choi-Williams Distribution (CWD) is given by:

$$
C_{f}(t, \omega, \phi)=\frac{1}{2 \pi} \iiint e^{j(\xi \mu-\tau \omega-\xi t)} \phi(\xi, \tau) A(\mu, \tau) d \mu d \tau d \xi
$$

Where $\varphi(\xi, \tau)$ is a kernel function and

$\mathrm{A}(\mu, \tau)=\mathrm{x}(\mu+\tau / 2) \mathrm{x}^{*}(\mu-\tau / 2)$

And $x(\mu)$ is time signal and $x^{*}(\mu)$ is its complex conjugate. This equation represents a generalized class of bilinear transformation that satisfies the marginal conditions and has good resolution in both the time and frequency spaces. The Wigner-Ville time-frequency distribution, is based on (7) where the kernel function is $\varphi$ $(\xi, \tau)=1$. For multi component signals the cross terms (Oscillatory positive and negative peaks, due to interference between spectral components) are present in the Wigner-Ville distribution. The cross terms cause interference that can obscure physically relevant components of the LPI signal's modulation ${ }^{[4]}$.

The Choi-Williams distribution uses an exponential weighting kernel in order to reduce the cross-term components of the distribution. The kernel function that gives the Choi-Williams distribution is:

$$
\phi(\xi, \tau)=e^{-\xi^{2} \tau^{2} / \sigma}
$$


Where $\sigma(\sigma>0)$ is a scaling factor. By substituting the above kernel equation (10) into (7), the equation for the discrete Choi- Williams distribution of the input signal $x(n)$ with a discrete time index and windowed for large data sample sets shown below:

$$
C W D_{x}(l, \omega)=2 \sum_{n=--N / 2}^{n=N / 2} S(l, n) e^{-j 2 \omega n}
$$

Where

$$
S(l, n)=W(n) \sum_{\mu=-M / 2}^{\mu=M / 2} \frac{1}{\sqrt{\frac{4 \pi n^{2}}{\sigma}}} e^{\frac{-(\mu-l)^{2}}{\frac{4 \pi \pi^{2}}{\sigma}}} x(\mu+n) x^{*}(\mu-n) W(\mu)
$$

Here $l$ is the time variable, $\omega$ is the angular frequency variable, $\sigma$ is a positive-valued scaling factor, and $*$ indicates the complex conjugate. and $W(n)$ is a symmetrical window (such as Hamming) which has nonzero values on the interval $-N / 2$ to $N / 2$ and $W(\mu)$ is a uniform rectangular window that has a value of one for the range of $-M / 2$ and $M / 2$. The choices of $N$ and $M$ on these windows respectively determine the frequency resolution of the CWD and the range at which the function will be defined ${ }^{[4-5]}$.

The discrete CWD can be modified to fit the standard discrete Fourier Transform (DFT) by setting $\omega=\pi \mathrm{k} / 2 \mathrm{~N}$. The final equation can be written as:

$$
C W D_{x}\left(l, \frac{\pi k}{2 N}\right)=2 \sum_{n=0}^{n=2 N-1} S^{\prime}(l, n) e^{-j 2 \pi k n / N}
$$

Where the kernel function $\mathrm{S}^{\prime}(l, n)$ is defined as:

$$
\begin{aligned}
S^{\prime}(l, n) & =S(l, n) & & 0 \leq n \leq N-1 \\
& =0 & & n=N \\
& =S(l, n-2 N) & & N+1 \leq n \leq 2 N-1
\end{aligned}
$$

\subsubsection{Reasons for choosing CWD}

1. The CWD of multi component signal achieves the cross term reduction.

2. The selection of appropriate window size, which depends on the kind of analyzed information, is not required for computation of CWD.

3. CWD satisfies the time marginal \& frequency marginal condition i.e. by summing the time frequency distributions over all frequencies one can obtain the instantaneous energy of the signal at a particular instance and by summing the time-frequency distributions over all time, one can obtain the power spectrum of the signal at a particular frequency.

4. Energy of the CWD is the same as the energy content in the signal ${ }^{[6]}$.

In the present paper, a four bit Frank Coded signal having 16 phases is considered. A carrier frequency $\mathrm{f}_{\mathrm{c}}=1 \mathrm{GHz}$ and the sampling frequency $\mathrm{fs}=2700 \mathrm{MHz}$ is used. Choi-Williams Distribution algorithm is applied on the given Frank coded signal sample and results of the CWD output are plotted against the time and frequency using the three dimensional mesh plot. Application of Choi-Williams Distribution algorithm on the multi component signal results in the time frequency graph as shown below.

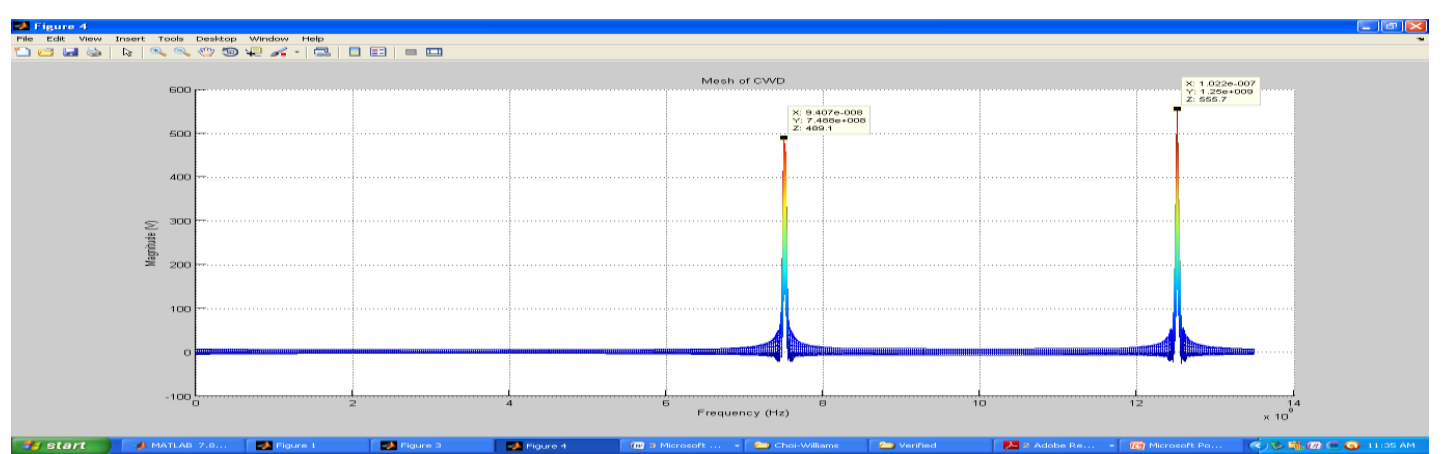

Figure 9: CWD output with no cross terms. 
Fig 9 shows the output of the CWD mesh plot when two frequency components $750 \mathrm{MHz}$ and $1250 \mathrm{MHz}$ are present in the signal. Due to the exponential kernel the cross terms are absent in the CWD.

The same signal when analyzed using Wigner-Ville distribution has one more frequency in plot at $1 \mathrm{GHz}$ which is a cross term as shown in the WVD contour plot in fig 10. Due to the exponential kernel the cross term is absent in the CWD.

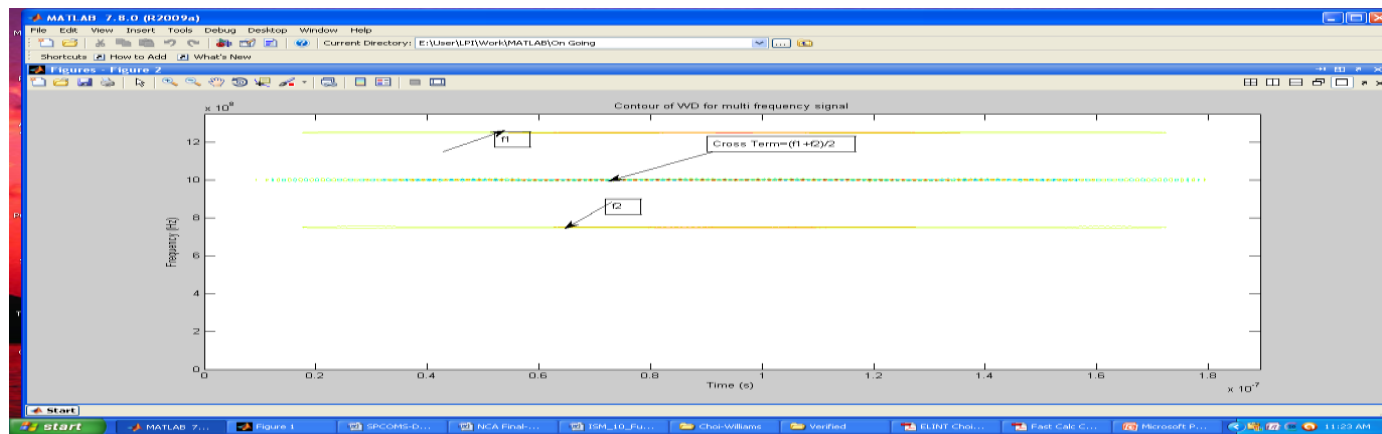

Figure 10: WVD output with cross term at $1 \mathrm{GHz}$.

The following plot shows the output of the CWD for a 4 bit Frank coded signal having a carrier frequency of $1 \mathrm{GHz}$ and Sampled at 2.7GSPS, with 16 phases over total Code duration of $32 \mathrm{nsec}$.

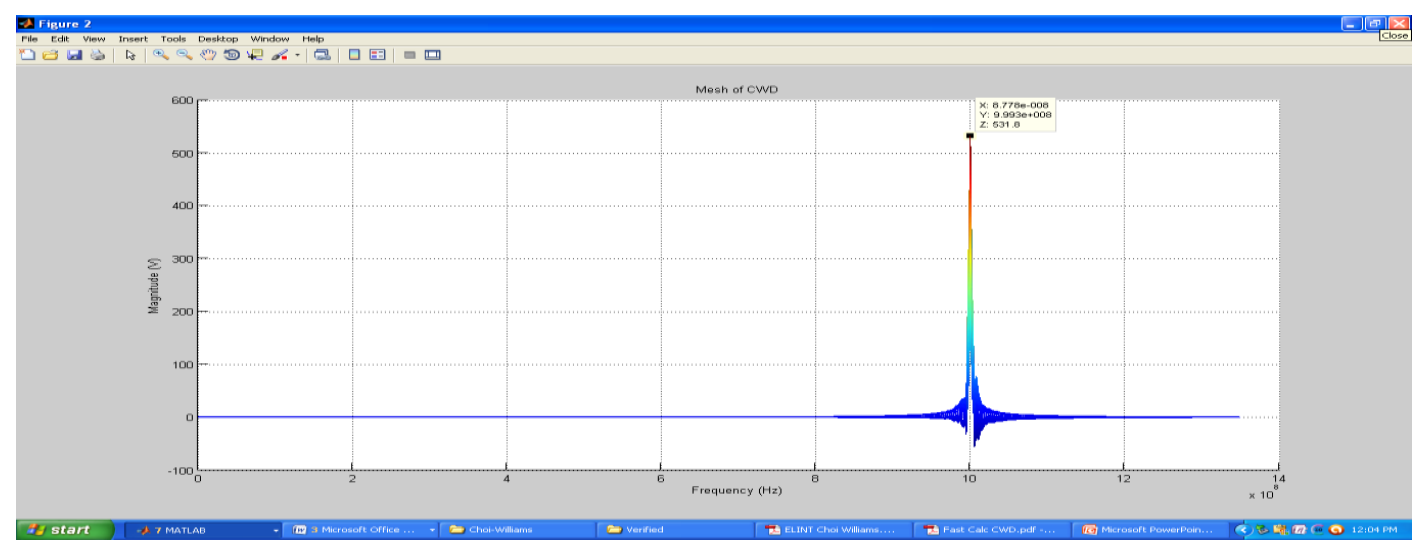

Figure 11: CWD mesh plot for extraction of Frequency.

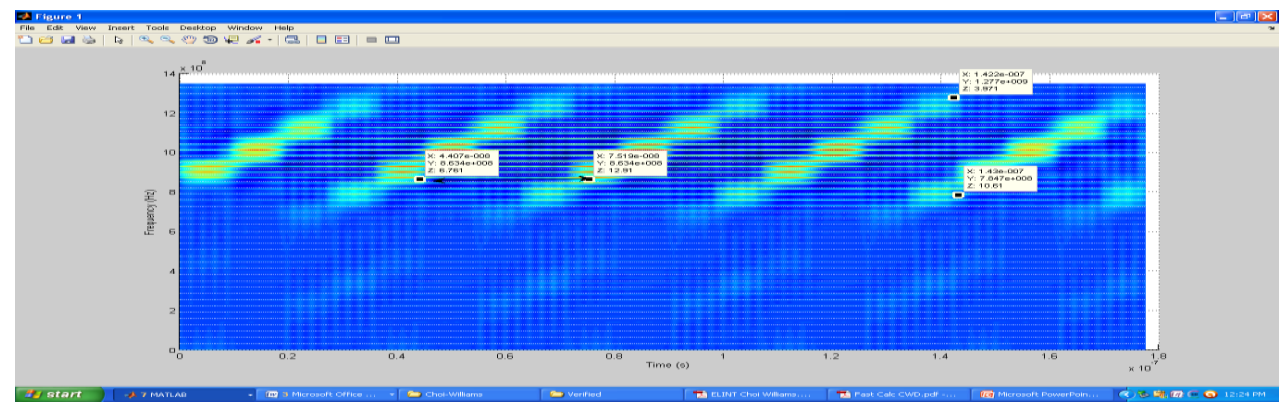

Figure 12: CWD mesh plot for extraction of Time Period and Bandwidth

The frequency of the signal can be found out from the peak of the mesh plot at view angle of $0^{0}$ elevation and $90^{\circ}$ in azimuth. From fig 10 we can observe that the frequency corresponding to the peak occurs at 999.3MHz. The horizontal distance between the successive strips on the mesh plot gives the total code duration (T) of the signal. From the fig 11, it can be observed as 75.19-44.07=31.12 ns. The bandwidth of the signal can be found out from the vertical distance between successive spectrogram strips. From the fig 11 the bandwidth is calculated as $1277-784=493 \mathrm{MHz}$. Once these parameters are known, the sub code duration $\left(\mathrm{t}_{\mathrm{b}}\right)$ can be calculated as the inverse of Bandwidth. The number of cycles of the carrier per phase (cpp) i.e. within each code duration, is given by the sub code duration $\left(t_{b}\right)$ multiplied by the frequency of the signal $\left(f_{c}\right)$ and converting the result to nearest integer. The no of phase changes are determined by dividing the total code duration $(\mathrm{T})$ by the sub code duration $\left(\mathrm{t}_{\mathrm{b}}\right)$ and converting to nearest integer ${ }^{[5]}$. 
The comparison of critical parameters obtained by CWD with the actual values of the parameters is shown in the below table.

TABLE 1

\begin{tabular}{|l|c|c|c|}
\hline Parameter & Actual Value & $\begin{array}{l}\text { Extracted } \\
\text { Value }\end{array}$ & Accuracy (\%) \\
\hline Frequency & $1 \mathrm{GHz}$ & $999.3 \mathrm{MHz}$ & 99.93 \\
\hline Total Code Duration & $32 \mathrm{nsec}$ & $31.12 \mathrm{nsec}$ & 97.25 \\
\hline Sub Code Duration & $2 \mathrm{nsec}$ & $2.0284 \mathrm{nsec}$ & 1.42 \\
\hline No of Phases & 16 & 16 & 100 \\
\hline $\begin{array}{l}\text { No of Cycles of the } \\
\text { Carrier }\end{array}$ & 2 & 2 & 100 \\
\hline
\end{tabular}

In the above results achieved the parameter detection accuracy of the algorithm depends on the number of input sample points $(\mathrm{N})$ used to calculate the CWD. If the $\mathrm{N}$ is increased, the more accuracy can be achieved at the cost of computational speed ${ }^{[6]}$. As a result of this property, the CWD is often thought as a signal's energy distribution in the time-frequency domain.

\section{Conclusion}

Six types of Low Probability of Intercept (LPI) signals were considered in this paper. They are Barker code, Frank code, and P1-P4 code signals. There are some of the signals generated for Choi-Williams Distribution analysis in this paper. The Choi-Williams Distribution is a two-dimension function describing the frequency content of a signal as a function of time. The Choi-Williams Distribution has been noted as one of the more useful time-frequency analysis techniques for signal processing. Using the Choi-Williams Distribution one can identify the frequency and time changes in most of the LPI signals.

\section{References}

[1] Philip E Pace, Detecting and Classifying LPI Radar, Second Edition, (Artech House, Inc., Norwood, Massachusetts, 2009).

[2] F.Taboada, A.Lima, J.Gau, P.Jarpa, and P.E.Pace, Intercept Receiver Signal Processing Techniques to Detect Low Probability of Intercept Radar Signals.

[3] Gau, J-Y, Analysis of Low Probability of Intercept Radar Signals using the Wigner Distribution, Naval Postgraduate School Master's Thesis, Sept. 2002.

[4] Aytuk Denk, Detection and Jamming Low Probability of Intercept (LPI) Radars, Naval Postgraduate School Master's Thesis, Sept. 2006.

[5] H.I.Choi and W.J.Williams, Improved time-frequency representation of multicomponent signals using exponential kernels: IEEE Trans. on Acoustics, Speech, and Signal Processing, vol. 37, no 6, pp. 862-871, 1989.

[6] L.Cohen, Time-frequency distributions: A review, Proc. IEEE, vol. 77, no 7, 1989, pp. 941-981. 